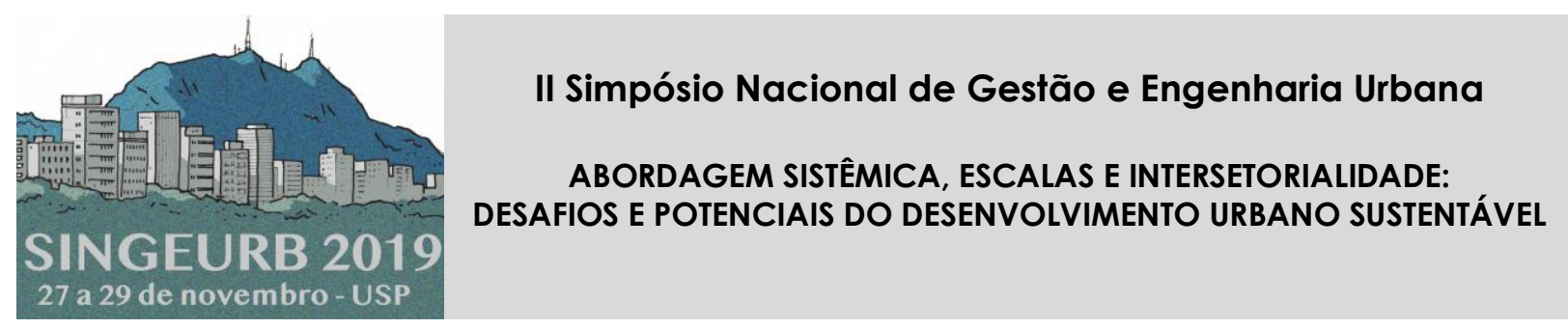

\title{
Documentos não gráficos no estudo da morfologia urbana: sistematização de informação em documentos históricos ${ }^{1}$
}

\section{Non-graphical documents in the study of urban morphology: information systematization of historical documents}

\author{
Corrêa, Rodolpho'; Pina, Silvia Mikami2; Monteiro, Evandro Z.3 \\ 1 Universidade Estadual de Campinas, R. Saturnino de Brito, 224 - Cidade \\ Universitária, Campinas - SP, 13083-889, Brasil, rodolpho.hcorrea@gmail.com \\ 2 Universidade Estadual de Campinas, silviaunicamp@gmail.com \\ 3 Universidade Estadual de Campinas, evanzigg@g.unicamp.br
}

\begin{abstract}
RESUMO
Um aspecto central para o estudo da morfologia urbana de uma região é o entendimento de como se deu sua ocupação e seu desenvolvimento. Tais estudos normalmente fazem uso de registros gráficos tais como mapas, plantas e bases cadastrais. Entretanto, nem sempre é possível dispor deste tipo de material, em especial no caso de cidades com ocupação antiga, pouco documentadas ou sem mapeamentos conhecidos. Diante disto, este trabalho busca identificar as possibilidades de utilização de registros não gráficos e de documentos oficiais, a partir do desenvolvimento e apresentação da metodologia utilizada para sistematizar os dados das Cartas de Datas de Terra da cidade de Campinas, entre 1815 e 1859. O estudo abrange os resultados parciais de pesquisa de mestrado em andamento que apresenta uma proposta metodológica para a utilização de documentos não gráficos para a produção de cartografias que supram a ausência de material gráfico contemporâneo ao desenvolvimento inicial das cidades, a partir de um estudo de caso. Os resultados obtidos até o momento indicam a viabilidade de se sistematizar e analisar os documentos de forma a localizar temporal e espacialmente os dados contidos nos registros escritos.
\end{abstract}

Palavras-chave: parcelamento, datas de terra, documentos não gráficos, método.

\begin{abstract}
A central point of the study of the urban morphology of a region is the understanding of how its occupation and its development took place. These studies usually make use of graphic records such as maps, plans and cadastral bases; however, it is not always possible to gather this type of material, especially in the case of old cities, poorly documented or without known mappings. Therefore, this present work seeks to identify the possibilities of using non-graphic records and official documents, from the development and presentation of the methodology used to systematize the data from "Cartas de Datas de Terra" of the city of Campinas, between 1815 and 1859. This present article encompasses part of the partial results of the master's project, currently under development, that seeks to generalize, from the case studied, a methodology that allows the use of non-graphic documents for the production of cartographies that overcomes the absence of contemporary graphic material for the initial

\footnotetext{
${ }^{1}$ CORRÊA, Rodolpho; PINA, Silvia Mikami; MONTEIRO, Evandro Z. Documentos não gráficos no estudo da morfologia urbana: sistematização de informação em documentos históricos. In: II SIMPÓSIO NACIONAL DE GESTÃo E ENGENHARIA URBANA: SINGEURB, 2019, São Paulo. Anais... Porto Alegre: ANTAC, 2019.
} 
development of the cities. The results hitherto obtained indicate the viability of systematizing and of analyzing the documents in order to locate the data contained in the records in a temporal and in a spatial manner.

Keywords: land parcel, urban morphology, non-graphycal document, method.

\section{INTRODUÇÃO}

O campo da morfologia urbana desenvolveu-se com contribuições de diferentes áreas do conhecimento, sendo especialmente relevantes os conceitos e procedimentos da geografia urbana. $O$ uso de mapas e plantas como base do estudo morfológico remonta ao trabalho de geógrafos alemães (GAUTHIEZ, 2004) no final do século XIX, adquirindo uma crescente importância e variedade nas formas de utilização, consoante as diferentes metodologias (OLIVEIRA, 2018, pp 9-11) utilizadas no estudo da forma urbana.

Mapas e plantas possuem a característica de relacionar, registrar e sintetizar visualmente uma série de elementos morfológicos e permitir explicitar e comparar as relações entre estes elementos. Se tomarmos um registro contemporâneo de uma região ou cidade eles permitirão identificar as relações atualmente válidas para a área, podendo permitir também a extrapolação de cenários futuros, fornecendo indícios das origens e do desenvolvimento daquela morfologia específica. Entretanto, no que tange aos processos históricos de formação, tais indícios só podem ser confirmados pela comparação de diferentes registros ao longo de uma série histórica relativamente abrangente.

Especialmente nestas áreas de ocupação antiga, um problema com que o investigador pode se deparar é a ausência ou insuficiência dos registros gráficos para a comparação ao longo de um período de tempo, seja pela inexistência de originais no caso de regiões pouco documentadas, seja pela não conservação destes registros. Nestes casos, a identificação e sistematização dos processos geradores da forma urbana podem ser prejudicados, na medida em que não haverá a disponibilidade de fontes para confirmar as hipóteses levantadas.

Assim o objetivo deste trabalho é identificar as potencialidades no uso de registros não gráficos, de textos oficiais, como base para suprir a inexistência de uma cartografia histórica adequada ao estudo da morfologia urbana, por meio de estudo realizado em Campinas-SP, a partir da coleta e sistematização de dados das "Cartas de Data de Terra", emitidas pela Câmara Municipal entre 1815 e 1859. Como resultado, uma proposta metodológica é apresentada para a utilização de tais documentos não gráficos para a produção de cartografias e, deste modo, indicar outras possibilidades cartográficas para cidades fundadas ainda no período colonial.

\section{O PARCELAMENTO DO SOLO E AS DOCUMENTAÇÕES OFICIAIS}

Para o entendimento do desenvolvimento da morfologia urbana de uma região é fundamental conhecer, conforme aponta Conzen (1960), as dinâmicas envolvidas na conversão de terras rurais ou devolutas em solo urbano e o sistema de parcelamento deste solo, o que, em certa medida, corresponde a acompanhar as mudanças na propriedade e na posse do solo e as alterações sofridas pelas parcelas.

Dentro do sistema jurídico medieval português, transplantado para a colônia na América, o crescimento inicial de uma vila estava diretamente associado à ocupação de seu rossio, uma área demarcada para utilização como campo comunal dos habitantes da vila, sob autoridade direta da câmara municipal (RIBEIRO, 2011) que tinha o poder de regular a abertura de novas ruas, demarcar as quadras (MOREIRA, 2003) e distribuir terras. Nesse sistema as terras de uso comum consideradas devolutas eram cedidas aos solicitantes na forma de "Datas de Terra", mediante algumas obrigações, como a de construir em um dado prazo, sob pena da parcela ser considerada novamente devoluta. As Câmaras mantinham registros destas operações de cessão - nas Cartas de Data de Terra - e de demarcação das parcelas - nos Termos de Arruação. 
Até a promulgação da Lei de Terras 2 em 1850, houve a passagem da terra comum para a parcela urbana por meio destas cessões. A lei de terras extinguiu esse sistema, convertendo as posses e cessões em propriedade plena e dando às Câmaras a propriedade das terras devolutas dos rossios, que passaram a ser transacionadas em hasta pública. A partir disto, os registros de datas de terra também declinaram, sendo substituídos pelos registros paroquiais e, posteriormente, pelo registro geral ${ }^{3}$ que, com as alterações sofridas pelos diferentes códigos ao longo do século XX, culminou no Registro de Imóveis como hoje é conhecido.

Há, desta forma, uma cronologia de documentos não gráficos, mantidos por órgãos oficiais que têm a potencialidade de acompanhar, ainda que com precisão variável, as mudanças na propriedade do solo urbano desde o final do período colonial até a atualidade.

\section{1 informações de interesse em documentos não gráficos}

Ao se trabalhar com fontes primárias de documentação, especialmente se tratando de registros não gráficos, as características das informações que se pode extrair variam conforme o tipo de documento disponível. A utilização de registros oficiais, notariais e cartoriais apresenta a vantagem de possuírem uma forma e um conteúdo relativamente estável e confiável ao longo de uma série temporal. A avaliação se um tipo de documento é útil para o estabelecimento de uma cartografia a partir de suas informações está associada à informação e a precisão que pode ser obtida e depende de alguns fatores:

- Inicialmente, considerando-se que cada registro trate de uma propriedade, ou de uma parcela, é preciso que haja uma série de documentos suficientemente grande, em quantidade e em distribuição temporal, de forma que a análise conjunta dos dados permita identificar a emergência de padrões específicos e suas transformações, separando-os dos casos singulares;

- O interesse de se produzir ou rastrear uma cartografia requer que as informações permitam localizar geograficamente a parcela, seja pela presença de um sistema de endereçamento ou um sistema de referências. A presença de indicações de dimensões, limites e propriedades confrontantes que possam ser associadas na série de dados auxiliam esta localização e permitem aumentar a precisão do dado obtido e

- Em se tratando de documentos oficiais de propriedade, quanto mais completas forem as qualificações civis do proprietário (como nome, estado civil, etc.) maiores as oportunidades de associação do registro com outros similares ou com outras séries de documentos, em um estudo mais amplo.

\section{COLETA E SISTEMATIZAÇÃO DAS INFORMAÇÕES: O CASO DE CAMPINAS-SP}

O município de Campinas encontra-se na situação referida de ausência de documentos gráficos contemporâneos ao seu estabelecimento e primeiras fases de desenvolvimento: a fundação de Campinas remonta ao ano de 1774 (PUPO, 1969) porém os primeiros mapas e plantas de cidade de que se tem notícia datam apenas do final do século XIX, sendo o mais antigo de 1878 e o primeiro levantamento rigoroso, feito sob encomenda do poder público municipal, datado de 1929 (Figura 1).

\footnotetext{
2 Lei no601, de 18/09/1850, regulamentada pelo Decreto ำ1.318, de 30/01/1854

${ }^{3}$ Lei no1.237, de 24/09/1864, regulamentada pelo Decreto no3.453, de 26/04/1865
} 
Figura 1 - Planta da Cidade de Campinas, folha n¹5, levantada em 1929

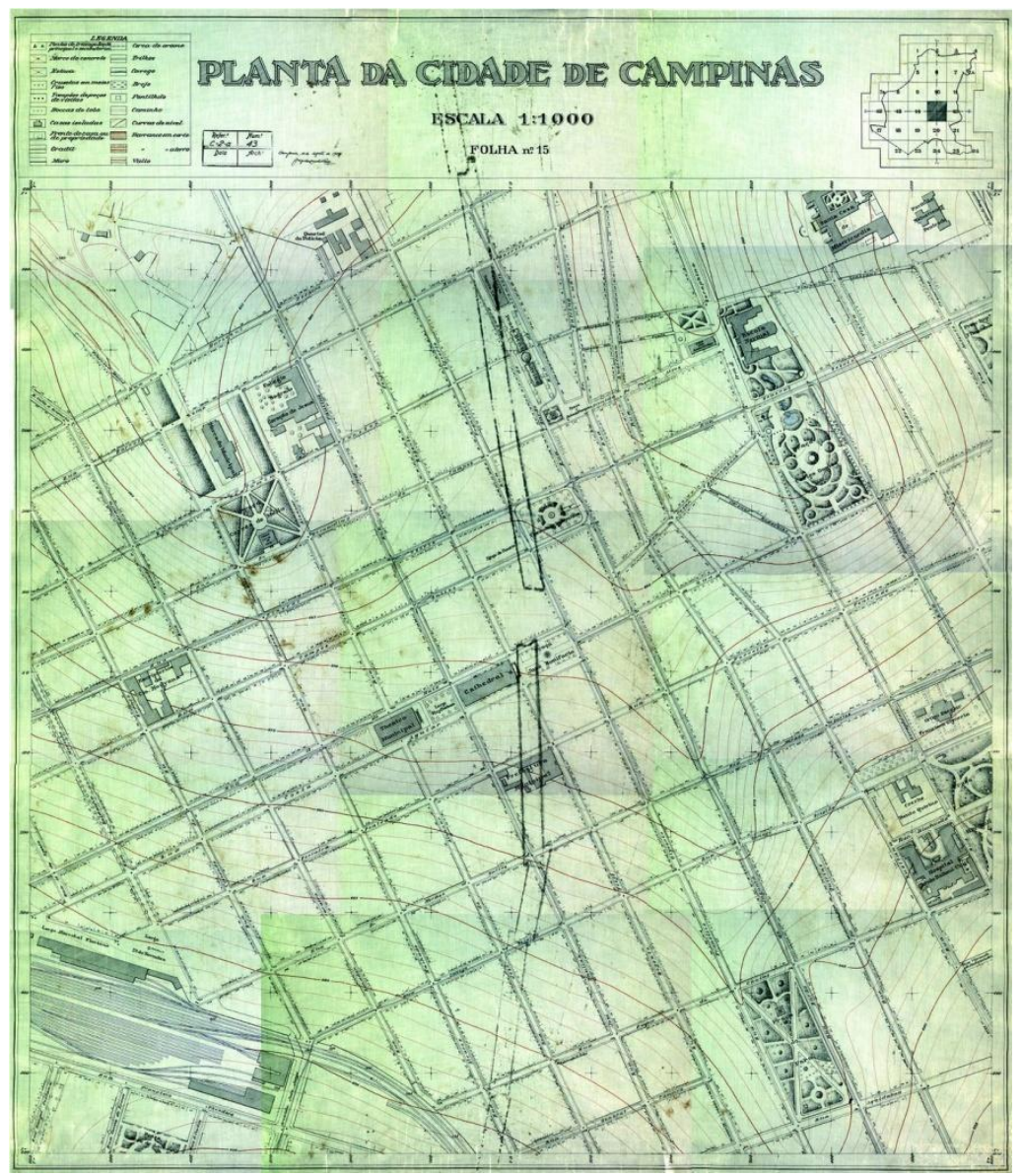

Fonte: Arquivo Municipal de Campinas

Entretanto, os registros originais de cessões das Cartas de Datas de Terra ainda são conservados pelo Arquivo da Câmara Municipal de Campinas (ACMC), na forma de dez volumes manuscritos abrangendo os anos de 1815 a 1859. Estes registros (Figura 2) contém a estabilidade necessária em sua estrutura para que os dados possam ser extraídos e comparados. Em que pese pequenas variações nas fórmulas jurídicas empregadas e no estilo do texto ao longo do período, cada volume é formado por um conjunto de Cartas de Datas de Terra sequencialmente registradas, sendo que cada registro individual contém:

- Um termo de abertura inicial contendo a requisição de uma data de terra por um dos habitantes da vila, já indicando o local no Rossio em que desejava obter a parcela;

- A descrição da deliberação da câmara favorável ao requerente, com a indicação da porção de terra a ser cedida, normalmente "seis por dezesseis braças de terra" (cerca de $13,20 \mathrm{~m} \times 35,20 \mathrm{~m})$;

- O termo de arruamento, registrado pelo arruador da câmara, contendo a localidade, as dimensões da parcela e as divisas e

- Um encerramento com as declarações de obrigações e direitos do requerente e a data do registro. 
Figura 2 - Data de terra concedida a Illario da Conceição

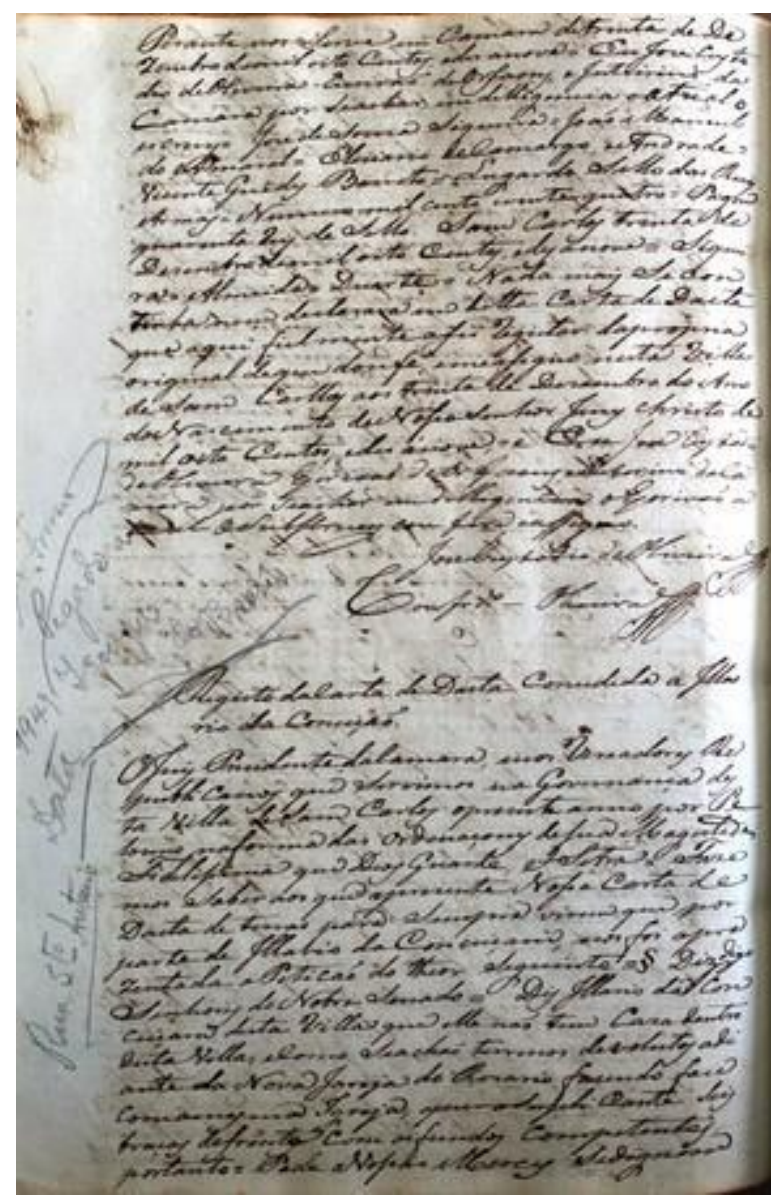

Fonte: Original pertencente ao Arquivo da Câmara Municipal de Campinas. Foto dos autores.

Tais registros foram lidos e seus dados principais transcritos e sistematizados em um banco de dados que totalizou 1612 entradas. O exemplo de uma linha típica deste banco (Quadro 1) contém as informações de relativas à data e localização do registro nos livros originais, o nome do requerente (chamado "suplicante" nas cartas), a localização da parcela conforme a petição feita à Câmara e suas dimensões e divisas.

Quadro 1 - Registro da data de terra de Illario da Conceição

\begin{tabular}{|c|c|c|c|c|c|c|c|}
\hline Livro & Folha & Data Reg. & Suplicante & Local Indicado & Ft & Fd & Divisas \\
\hline 23 & $\begin{array}{c}46 \\
\text { verso }\end{array}$ & $30 / 12 / 1819$ & $\begin{array}{c}\text { Illarioda } \\
\text { Conceição }\end{array}$ & $\begin{array}{c}\text { "Rua de Santo Antônio } \\
\text { adiante da nova igreja do } \\
\text { Rosário fazendo face coma } \\
\text { mesma" }\end{array}$ & 6 & 16 & $\begin{array}{c}\text { "divisando na qual } \\
\text { parte com terras } \\
\text { devolutas, para a } \\
\text { direita,e a esquerda } \\
\text { com Francisco Teixeira" }\end{array}$ \\
\hline
\end{tabular}

Fonte: Dados extraídos pelos autores a partir do registro constante no Tomo n 23 "Termos de arruação e posse de cartas de data de terra da Câmara da Vila de São Carlos, 1817-1821" do Arquivo da Câmara Municipal de Campinas.

Embora o local indicado não corresponda a um endereço completo 4 uma vez estabelecido um banco de dados com tais informações, é possível comparar os diferentes registros relacionando entre si as nomenclaturas populares das vias e as divisas das parcelas, formando redes de vizinhança que podem ser ordenadas e representadas na forma de grafos (Figura

\footnotetext{
${ }^{4}$ Campinas só estabeleceu uma denominação oficial para as ruas em 1848.
} 
3), de modo que, se for possível localizar uma das parcelas da rede, as demais relacionadas podem ser localizadas também, aumentando a precisão dos dados.

Figura 3 - Detalhe dos Grafos de relações de vizinhança entre as datas de terra

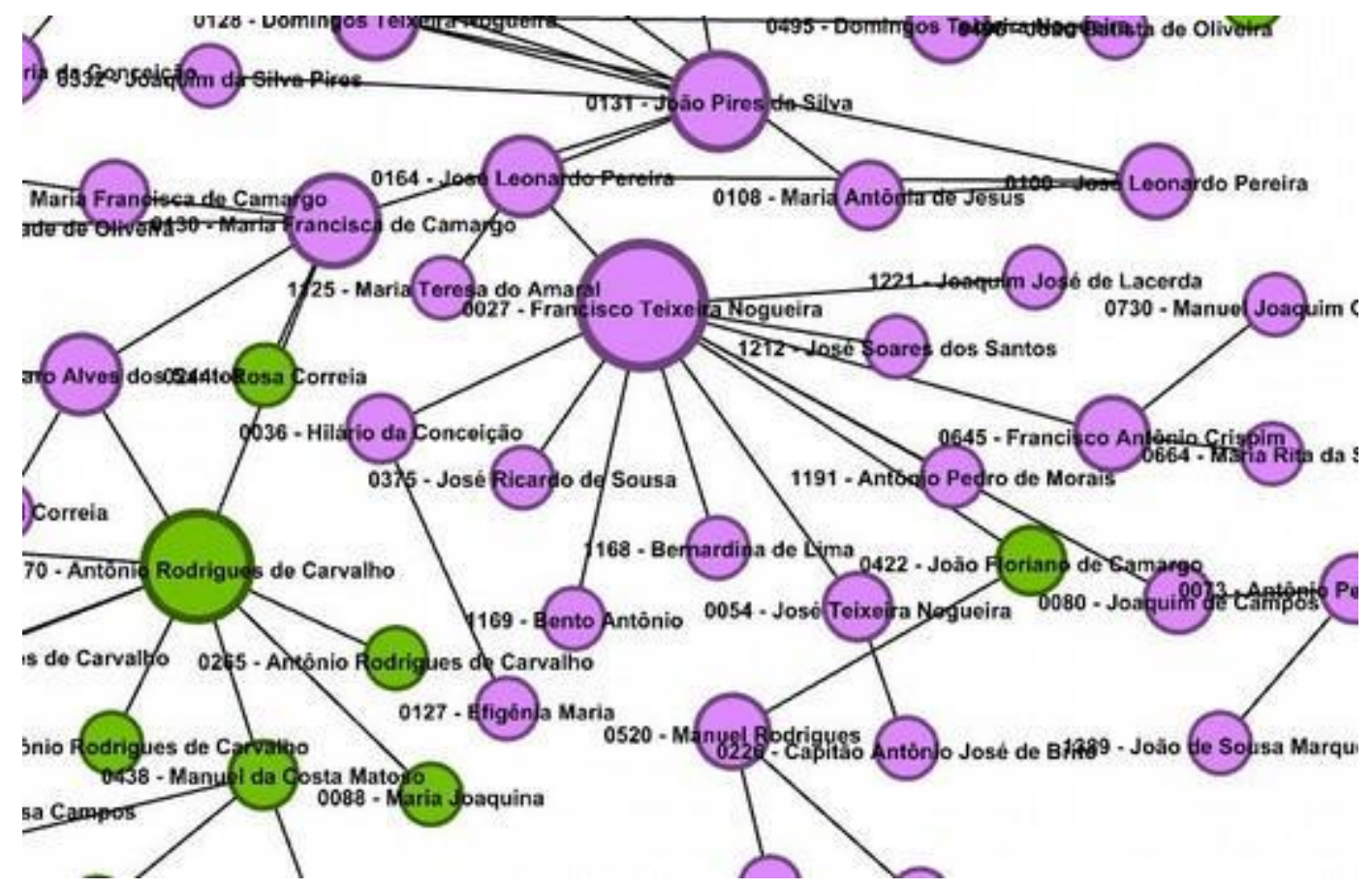

Fonte: Os autores

As indicações de localização registradas nestes documentos, comparadas com as denominações oficiais das vias empregadas posteriormente, permite identificar os caminhos principais, os marcos utilizados como pontos de referências e todo o sistema de orientação dos habitantes. Neste aspecto, a ausência de um sistema de endereços e de numeração oficial acaba por revelar dinâmicas sociais que vão se refletir na morfologia urbana do local, na medida em que os requerentes, ao solicitarem terras em certas regiões em detrimento de outras, revelam os eixos preferenciais de crescimento e as regiões do rossio mais valorizadas e primeiramente ocupadas (Figura 4).

Figura 4 - Dispersão das Datas de Terra concedidas em Campinas, por ano e por região do Rossio

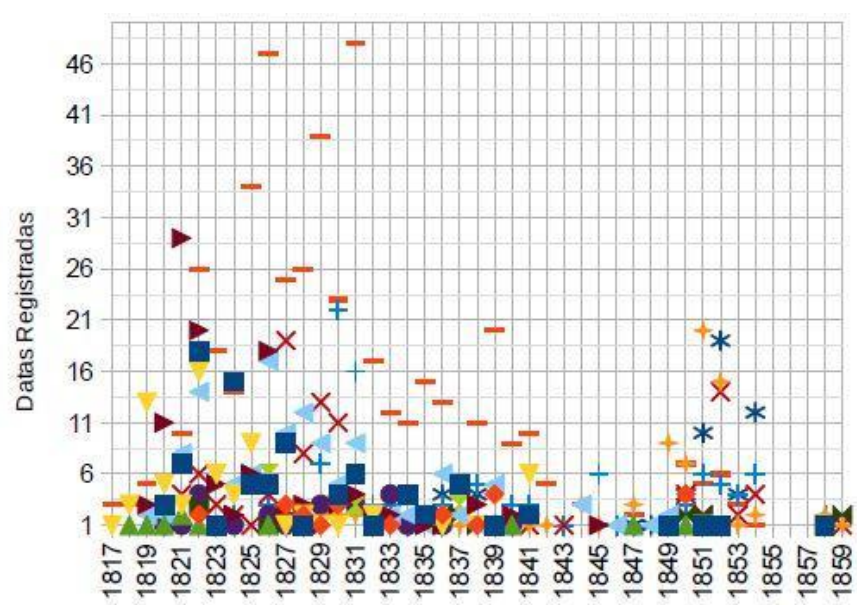

Ano
Bairro Alto

- Campinas Velhas

* Santa Cruz

A Matriz Velha

- Rosário

4 Matriz Nova

Mrejo do Nascente

x Brejo do Poente

- Cemitério Velho

+ Campo

$\times$ Saída de Itu

+ Saída de São Paulo

* Cambuizal

- Não Explicitado

Fonte: Os autores 


\section{CONCLUSÕES}

A partir do método exposto, se pode concluir que, diante da ausência de documentos gráficos - como mapas e plantas - em cidades de ocupação antiga, a utilização de registros oficiais não gráficos pode contribuir para a elucidação do desenvolvimento e ocupação do solo nos estágios iniciais de formação de uma cidade. Para isto, é necessário que haja um conjunto de dados que permita a comparação sistemática entre os registros, fazendo emergir padrões de ocupação, sistemas de orientação e eixos principais de desenvolvimento. Também é preciso que os registros apresentem uma estrutura uniforme o suficiente e forneçam dados que permitam localizar no tempo e no espaço da cidade as parcelas a que se referem. Conclui-se que a utilização de grandes séries de documentos oficiais relacionados a propriedade do solo, como são as Cartas de Data de Terra, têm o potencial de fornecer as informações necessárias e permitir a construção de suas cartografias.

\section{REFERÊNCIAS}

CONZEN, M. R. G. Alnwick, Northumberland: a study in town-plan analysis. Publication of the: Institute of British Geographers, Londres, n.27, pp.1-122, 1960.

GAUTHIEZ, B. The history of urban morphology. Urban Morphology, v.8.2, p.71-89, 2004.

Disponível em: <http://www.urbanform.org/online_public/2004_2.shtml>. Acesso em 28 ago. 2017

MOREIRA, R. A arte de ruação e a cidade luso-brasileira. Cadernos de pesquisa do LAP, $\mathrm{n}^{\circ}$ 37, p.8-30, jan-jun 2003.

OLIVEIRA, V. Diferentes abordagens em morfologia urbana: Contributos lUso-brasileiros. ISUF, 2018. Disponível em: <http://vitoroliveira.fe.up.pt/uf-ebooks> Acesso em 23 fev. 2018

PUPO, Celso Maria de Mello. Campinas, seu berço e juventude. Campinas: Academia Campinense de Letras, 1969.

RIBEIRO, Fernando V. Aguiar. A terra urbana colonial: o exemplo da vila de São Paulo. Anais (Eletrônicos) do XXVI Simpósio Nacional de História - ANPUH, São Paulo: 2011. Disponível em: <http://www.snh201 1.anpuh.org/conteudo/view?ID_CONTEUDO=775>. Acesso em: 24 set. 2018. 